\title{
A patient with abnormalities of the coronary arteries and non-compaction of the left ventricular myocardium resulting in ischaemic heart disease symptoms
}

\author{
J. Dąbek, M. Majewski, W. Walkowicz, Z. Gąsior \\ Chair and Department of Cardiology, School of Health Sciences, Medical University of Silesia, Katowice, Poland
}

[Received 4 February 2015; Accepted 26 February 2015]

\begin{abstract}
Left ventricular non-compaction ( $L V N C$ ) is a rare cardiomyopathy that results from unsettled embryogenesis of myocardium. It is morphologically characterised by the presence of non-compacted, this is hypertrabeculated, myocardium of the left ventricle with deep endocardial recesses. The clinical spectrum of symptoms is very wide - from asymptomatic patients through the cases of heart failure to the patients requiring heart transplantation. The diagnosis is most frequently based on the echocardiography. LVNC is often coexisted with other heart defects and coronary artery abnormalities. We described a case of a 58-year-old man with LVNC and coronary artery anomalies. (Folia Morphol 2015; 74, 4: 518-523)
\end{abstract}

Key words: cardiomyopathy, coronary artery abnormalities, myocardium, non-compaction

\section{INTRODUCTION}

Non-compaction of left ventricular myocardium (LVNC) is a rare, underdiagnosed, congenital cardiomyopathy resulting from an arrest of the endomyocardial morphogenesis during embryonic period. Cardiomyopathy is anatomically characterised by deep trabeculations in the ventricular wall which define recesses communicating with main ventricular cavity. Because of its spongy-like structure is sometimes named 'spongy cardiomyopathy'. The aetiology of that heart condition is not clear. Major clinical correlates are heart failure (HF), arrhythmias and thromboembolic events. Making diagnosis is based on the imaging modalities, especially echocardiography, and is often accidental. LVNC is frequently coexisted with other heart abnormalities as well as neuromuscular disorders.

\section{CASE REPORT}

58-year-old patient with a history of ischaemic heart disease (currently complaints in CCS class II), arterial hypertension, periodically occurring palpitations and lipid disorders was admitted to the Department of Cardiology in order to perform coronarography and to determine the optimal medical treatment.

Familiar history did not reveal anything significant. The patient had administered following medications: bisoprolol $5 \mathrm{mg}$ once daily, ramipril $5 \mathrm{mg}$ twice daily, trimetazidine $35 \mathrm{mg}$ twice daily, atorvastatin $20 \mathrm{mg}$ once daily and acetylsalicylic acid $75 \mathrm{mg}$ once daily.

On admission, the patient's general condition was good. On physical examination there were no relevant abnormalities. Heart rate was regular, 60/min, with a discreet holosystolic murmur. Blood pressure was 130/80 mm Hg.

Address for correspondence: J. Dąbek, Associated Professor, Chair and Department of Cardiology, School of Health Sciences, Medical University of Silesia, ul. Ziołowa 45/47, 40-635 Katowice-Ochojec, Poland, tel: +48 32359 85 30, e-mail: jdabek@sum.edu.pl 
An electrocardiogram (ECG) on admission revealed sinus rhythm, left axis deviation with incomplete left bundle branch block (QRS $=110 \mathrm{~ms}$ ), deep QS complexes in V1-V2 leads, lack of R-wave progression in the precordial leads and features of left ventricular hypertrophy with repolarisation changes.

In laboratory tests elevated level of total cholesterol and low density lipoprotein cholesterol $(228 \mathrm{mg} /$ $\mathrm{dL}$ and $136 \mathrm{mg} / \mathrm{dL}$, respectively) were found. Cardiac markers both on admission and in the control were negative.

Transthoracic echocardiography (TTE) revealed enlarged diameter of left atrium (42 mm), left ventricular ejection fraction (LVEF) $50 \%$ and hypokinetic basal segments of anterior wall. In the apical projection of the left ventricle, typical for non-compaction, two-layered structure of the myocardium with hypertrabeculated inner layer and deep recesses with blood contrasting visible using Doppler colour technique (Figs. 1, 2). Moreover, moderate mitral, tricuspid and pulmonary valve regurgitation were revealed.

Holter ECG monitoring revealed sinus rhythm with a mean frequency of $60 / \mathrm{min}$, numerous premature supraventricular contractions (1329/day) and 113 premature ventricular beats (VEs) including one episode of sustained ventricular tachycardia and two pairs of VEs.

Because of clinical presentation and results of additional tests the patient was enrolled for invasive diagnostics of coronary artery disease. Coronary angiography was performed and revealed anomaly of coronary arteries with the circumflex artery ( $C x-$ circumflex branch of left coronary artery) originating from the proximal segment of the right coronary artery (RCA) resulting in difficulties of its selective catheterisation. The left coronary artery (LCA) system revealed anterior descending artery (LAD - anterior interventricular branch of left coronary artery) without left main coronary artery (LMCA - clinical designation of initial segment of left coronary artery before its bifurcation into LAD and Cx) (Figs. 3, 4).

Due to the doubts regarding the epicardial coronary artery layout, computed tomography (CT) angiography was performed. CT imaging confirmed that $L A D$, not narrowed, leads directly from the left coronary sinus (no left main present) and Cx artery extends from the proximal part of large, not narrowed RCA, back from the aorta between it and left atrium (Fig. 5).

After diagnostic procedures the continuation of medical treatment and use of the following medica-

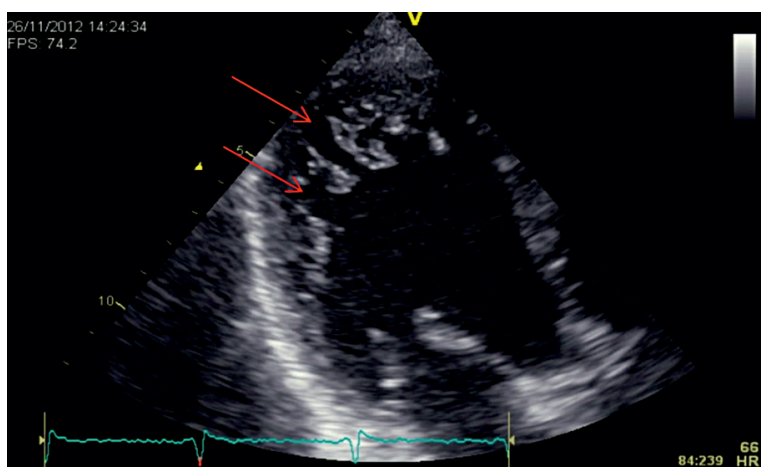

Figure 1. Transthoracic echocardiography of patient with left ventricular non-compaction. Double-layered, foetal-like structure of the left ventricular myocardium with hypertrabeculated inner layer and deep recesses (red arrows).

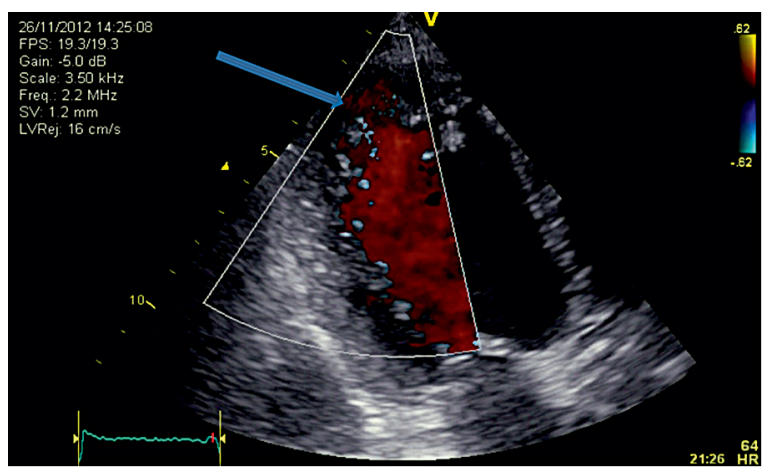

Figure 2. Colour Doppler echocardiography. Blood contrasting in the recesses of the left ventricular wall confirming anatomical connection with the left ventricle cavity (blue arrow).

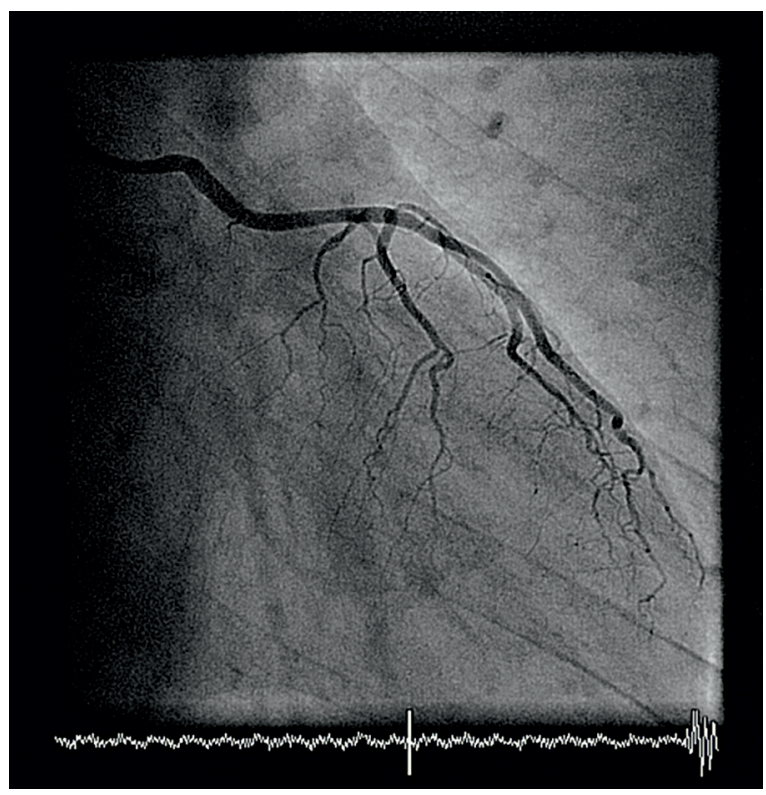

Figure 3. Coronary angiography. The left coronary artery system left anterior descending artery with no left circumflex artery visible. 


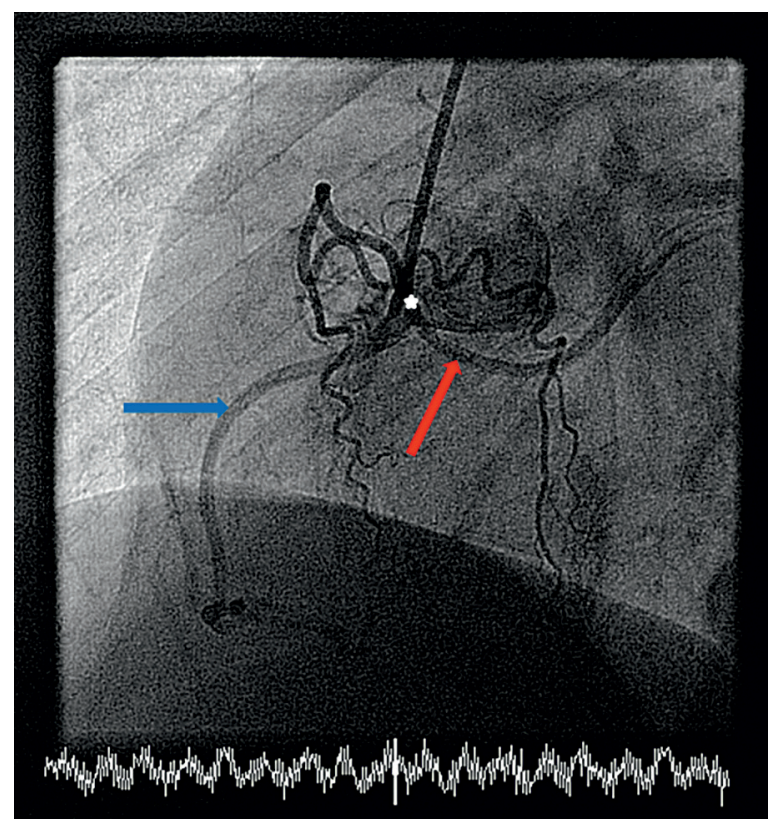

Figure 4. Coronary arteries seen after catheterisation and contrast injection to right coronary sinus. Big, not narrowed right coronary artery (blue arrow) and commencing from its proximal segment circumflex artery (red arrow). Asterisk indicates right coronary sinus.

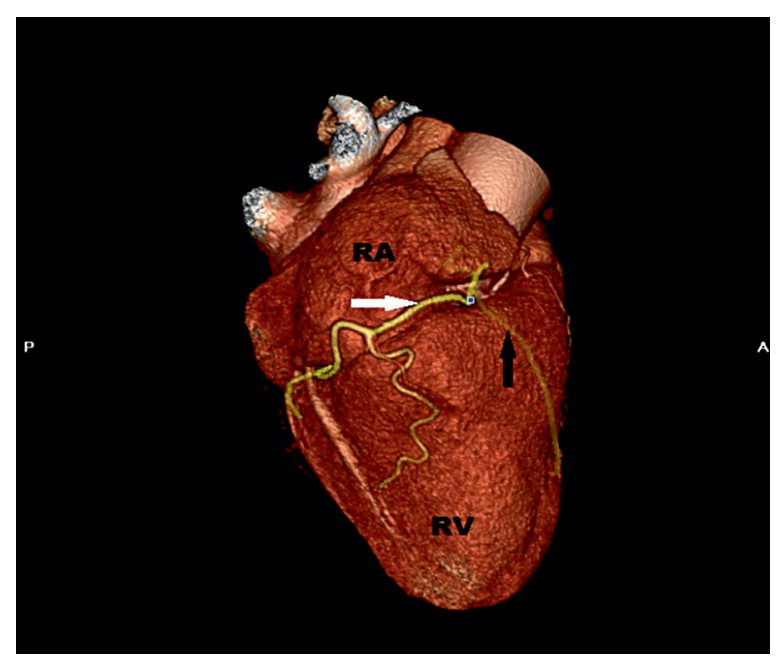

Figure 5. The system of epicardial arteries of patient with a non-compaction cardiomyopathy and coexisting anomaly of the coronary arteries layout obtained in computed tomography angiography. Right-sided projection. Large and not occluded right coronary artery (RCA, white arrow) and circumflex artery — branched out from the proximal part of RCA (black arrow) are visible; RA — right atrium; RV — right ventricle.

tions was administered: sotalol $80 \mathrm{mg}$ 1-0-1 (due to the occurrence of ventricular arrhythmias), trimetazidine $35 \mathrm{mg}$ 1-0-1, ramipril $5 \mathrm{mg}$ 1-0-1, atorvastatin $20 \mathrm{mg} \mathrm{0-0-1}$ and low-dose of acetylsalicylic acid $75 \mathrm{mg}$ daily. Periodical control visits in the Cardiology Outpatient Clinic were advised also.

\section{DISCUSSION}

The term 'isolated non-compaction of the left ventricular myocardium' was officially introduced into clinical practice by Chin et al. in 1990 [3] although the phenomenon of foetal phenotype myocardium in adults had been known much earlier.

The exact prevalence of LVNC is not known. Initially it was considered as a rare condition, but nowadays due to the wide using of imaging techniques, is recognised more frequently and often accidentally when patients undergo cardiovascular investigation for other reasons. According to the literature the prevalence of non-compacted cardiomyopathy in the general population ranges from $0.014 \%$ to $1.3 \%[10$, 14]. However it seems that the prevalence of LVNC may be underestimated because of its occurrence also in patients without clinical symptoms or because of subjective assessment of echocardiography findings. In the Australian study on the prevalence of primary cardiomyopathies in paediatric population LVNC accounted for $9.2 \%$ of cases and was the third most common cardiomyopathy beyond the dilated and hypertrophic cardiomyopathy [12].

The aetiology of LVNC is currently considered to be heterogeneously genetically determined. Several genes that may be responsible for the disease development has been characterised (e.g. genes of mitochondrial, cytoskeletal or sarcomeric proteins) [17], but a small number of data and uncertain relationship between genotype and phenotype require further investigation on that area. Myocardium has meshwork-like structure in prenatal period consisting of fine muscle trabeculae and recesses between them through which the myocardium is supplied directly by the blood stream from the heart cavity. Between 5 and 8 gestation week the myocardium undergoes reconstruction and compaction process. The process continues from epicardial to endocardial layer, from the base to the apex of the heart and from the septum to the free wall what is reflected in the localisation of non-compacted muscle in adults. Along with the compaction progress the development of coronary circulation continues. Abnormalities in coincidence of these processes explain frequent occurrence of coronary artery anomalies in case of LVNC. LVNC morphologically resembles heart muscle stopped at foetal period of development and embrace a continuum of morphological features ranging from normal appearance to the characteristic for fully-expressed LVNC cases that is determined by the moment of stopping 
normal development of the heart muscle compaction (the earlier period of development's arrest the larger non-compaction of myocardium).

There are no specific for LVNC histological changes, although fibrosis has been described in many reports $[4,5]$. It is worth to notice that isolated noncompaction may histologically differ from noncompaction associated with other congenital heart diseases in that the deep intertrabecular recesses communicate with the left ventricular cavity in the former and both the coronary circulation and left ventricle in the latter [14]. Burke et al. [2] showed in case series $(n=14)$ endocardial fibroelastosis was a characteristic histological feature, as well as anastomosing or polypoid endocardial trabeculations which resulted in staghorn-shaped, endocardial-lined recesses. However, more studies are needed to investigate histological features of LVNC.

Also the spectrum of clinical symptoms in patients with LVNC can be very wide - from asymptomatic cases to the patients requiring heart transplantation. Clinical presentation depends mainly on whether LVNC is an isolated defect or coexists with other congenital conditions or genetic syndromes, time of diagnosis: in childhood or adulthood, its circumstances: diagnosis in symptomatic patients or as screening procedures in relatives of patients with LVNC. Typical symptoms of cardiomyopathy are HF complaints, arrhythmias and thromboembolic events. It seems that the clinical presentation results not from the non-compaction itself but rather from presence of non-compaction associated with end-stage $\mathrm{HF}$ as a result of LVNC. Among cardiac arrhythmias, ventricular arrhythmias occur most often, but atrial fibrillation or Wolf-Parkinson-White syndrome could be also frequently present. The changes observed in the ECG are not specific and beside arrhythmias and conduction disturbances they could encompass left ventricular hypertrophy with repolarisation changes. Left bundle branch block (LBBB) is quite common finding in LVNC patients and could indicate for more advanced heart defects, but it is speculative if LBBB is a prognostic factor [19].

The diagnosis is based mainly on non-invasive imaging, especially TTE, and especially in doubtful cases magnetic resonance imaging (MRI) or CT could be of help. The most common echocardiographic criteria for LVNC diagnosis are those proposed by Jenni et al. [7] including:

- thickened myocardium with double-layered structure and the ratio of non-compacted to compac- ted layer $>2$, evaluated in end-systolic phase in short-axis projection;

- presence of deep recesses within the myocardium with the blood flow from the left ventricle visible in colour Doppler echocardiography technique;

- localisation of changes usually in the middle segments of free and inferior wall of the left ventricle as well as in the apex;

- lack of coexisting cardiac pathology.

It is worth noting that echocardiography is a quite subjective diagnostic method and the compliance of diagnosis by different echocardiographic criteria is quite poor, around $30 \%$ [9].

Initially, the spongy cardiomyopathy was considered as very severe condition with a poor prognosis which resulted from a late diagnosis of patients in advanced stage of a disease and with severe complaints. Current data suggest better outcomes, particularly when the disease is asymptomatic or early recognised $[10,11,14,16]$. Conditions deteriorating the prognosis embrace significant advancement of $\mathrm{HF}$ symptoms (III or IV NYHA class), sustained ventricular tachycardia and advanced age. Some echocardiographic features such as large number of occupied segments, left ventricular end-diastolic diameter or even the ratio of non-compacted to compacted layer may also worsen the prognosis [13]. The most common complications include HF, ventricular arrhythmias, thromboembolic events and sudden cardiac death. In over $20 \%$ of patients with advanced stage of disease there are life-threatening ventricular arrhythmias inherent $[4,18]$. Prospective French study involving a group of patients with LVNC showed that in the case of people who had LVNC detected accidentally or as a part of the screening procedure of burdened relatives outcomes are quite good, but on the other hand $\mathrm{HF}$ or left ventricular dysfunction were associated with a higher risk of death and need of heart transplant [6]. No changes in the ECG are associated with a better prognosis [15].

Treatment of patients with LVNC due to the lack of any causal therapy is concentrated on the major complications of the disease: $\mathrm{HF}$, arrhythmias, prevention of sudden cardiac death and thromboembolic events. In patients with congestive HF, standard, guidelines-based treatment should be administered. There is some evidence that early initiation of typical HF treatment in LVNC patients with or without clinical symptoms, especially in the beginning of left ventricular dysfunction, could be beneficial and delay 
fully-expressed HF clinical presentation [2]. Some authors have demonstrated that cardiac resynchronisation therapy can results in improvement of left ventricular function in patients with a dilated left ventricle coexisting with LVNC [1]. Due to the high risk of sudden cardiac death caused by arrhythmias an implantation of cardioverter-defibrillator device (ICD) based on conventional criteria used in HF should be always considered. Kobza et al. [8] demonstrated the validity and effectiveness of ICD implantation in both primary and secondary prevention of sudden cardiac death in patients with LVNC. Most controversial issue associated with LVNC therapy is anticoagulation treatment. LVNC is associated with significantly higher risk of thromboembolic complications. Structure of non-compacted myocardium predisposes to formation of blood clots in deep recesses as a result of slow blood flow and subendocardial fibrosis. It seems, however, that the incidence of thromboembolic complications in previous studies was overestimated due to more advanced stage of disease which resulted in recommendation of anticoagulation in every patient [14]. Currently the risk of thromboembolic events is considered to be much lower and decision if anticoagulation therapy should be administered must be made individually taking into account clinical features increasing risk of thrombotic complications such as: impaired left ventricular systolic function, atrial fibrillation, previous embolic incidents or presence of thrombus in the left ventricle. Some data indicate aspirin could be effective in preventing thromboembolic complications. In paediatric patients taking aspirin since LVNC diagnosis was made, no thromboembolic complications in long-term follow-up were observed [11].

It is also worth to notice that some aetiopathogenetic features of LVNC may also accelerate the development of atherosclerosis [20]. In case of late diagnosis of LVNC, coronary insufficiency symptoms may be first complaints, as in described patient.

\section{CONCLUSIONS}

Summarising: the coincidence of coronary artery system's anomalies with spongy cardiomyopathy, causing difficulties in the interpretation of diagnostic imaging and possibility of complications, requires peculiar attention during performing coronarography and percutaneous coronary interventions. In the above-mentioned case the patient's complaints of chest pain suggesting coronary artery disease when there were no narrowing of coronary vessels in coronarography suggests their source in abnormal myocardium blood supply caused by the lack of left main coronary artery and its branch - circumflex artery - originating from the initial segment of RCA as well as remnants of abnormal, foetal-like, non-compacted heart muscle structure.

\section{REFERENCES}

1. Bertini M, Ziacchi M, Biffi M, Biagini E, Rocchi G, Martignani C, Ferlito M, Pasquale F, Cervi E, Branzi A, Rapezzi C, Boriani G (2011) Effects of cardiac resynchronisation therapy on dilated cardiomyopathy with isolated ventricular non-compaction. Heart, 97: 295-300. doi: 10.1136/ /hrt.2010.211607.

2. Burke A, Mont E, Kutys R, Virmani R (2005) Left ventricular noncompaction: a pathological study of 14 cases. Hum Pathol, 36: 403-411.

3. Chin TK, Perloff JK, Williams RG, Jue K, Mohrmann R (1990) Isolated noncompaction of left ventricular myocardium. A study of eight cases. Circulation, 82: 507-513.

4. Conraads V, Paelinck B, Vorlat A, Goethals M, Jacobs W, Vrints C (2001) Isolated non-compaction of the left ventricle: a rare indication for transplantation. J Heart Lung Transplant, 20: 904-907.

5. Finsterer J, Stollberger C, Feichtinger H (2002) Histological appearance of left ventricular hypertrabeculation/ noncompaction. Cardiology, 98: 162-164.

6. Habib G, Charron P, Eicher JC, Giorgi R, Donal E, Laperche T, Boulmier D, Pascal C, Logeart D, Jondeau G, Cohen-Solal A (2011); Working Groups 'Heart Failure and Cardiomyopathies' and 'Echocardiography' of the French Society of Cardiology. Isolated left ventricular non-compaction in adults: clinical and echocardiographic features in 105 patients. Results from a French registry. Eur J. Heart Fail, 13: 177-185. doi: 10.1093/eurjhf/hfq225.

7. Jenni R, Oechslin E, Schneider J, Attenhofer Jost C, Kaufmann PA (2001) Echocardiographic and pathoanatomical characteristics of isolated left ventricular non-compaction: a step towards classification as a distinct cardiomyopathy. Heart, 86: 666-671.

8. Kobza R, Jenni R, Erne P, Oechslin E, Duru F (2008) Implantable cardioverter-defibrillators in patients with left ventricular noncompaction. Pacing Clin Electrophysiol, 31: 461-467. doi: 10.1111/j.1540-8159.2008.01015.x

9. Kohli SK, Pantazis AA, Shah JS, Adeyemi B, Jackson G, McKenna WJ, Sharma S, Elliott PM (2008) Diagnosis of left-ventricular non-compaction in patients with left-ventricular systolic dysfunction: time for a reappraisal of diagnostic criteria. Eur Heart J, 29: 89-95. doi: 10.1093/eurheartj/ehm481.

10. Lilje C, Razek V, Joyce JJ, Rau T, Finckh BF, Weiss F, Habermann CR, Rice JC, Weil J (2006) Complications of non-compaction of the left ventricular myocardium in a paediatric population: a prospective study. Eur Heart J, 27: 1855-1860. doi: 10.1093/eurheartj/ehl112.

11. Murphy RT, Thaman R, Blanes JG, Ward D, Sevdalis E, Papra E, Kiotsekoglou A, Tome MT, Pellerin D, McKenna WJ, Elliott PM (2005) Natural history and familial characteristics of isolated left ventricular non-compaction. Eur Heart J, 26: 187-192. doi: 10.1093/eurheartj/ehi025. 
12. Nugent AW1, Daubeney PE, Chondros P, Carlin JB, Cheung M, Wilkinson LC, Davis AM, Kahler SG, Chow CW, Wilkinson JL, Weintraub RG (2003) National Australian Childhood Cardiomyopathy Study. The epidemiology of childhood cardiomyopathy in Australia. N Engl J Med, 348: 1639-1646. doi: 10.1056/NEJMoa021737.

13. Oechslin E, Jenni R (2011) Left ventricular non-compaction revisited: a distinct phenotype with genetic heterogeneity? Eur Heart J, 32: 1446-1456. doi: 10.1093/eurheartj/ lehq508.

14. Oechslin EN, Attenhofer Jost CH, Rojas JR, Kaufmann PA, Jenni R (2000) Long-term follow-up of 34 adults with isolated left ventricular noncompaction: a distinct cardiomyopathy with poor prognosis. J Am Coll Cardiol, 36: 493-500.

15. Peters F, Khandheria BK (2012) Isolated left ventricular noncompaction: what do we really know? Curr Cardiol Rep, 14: 381-388. doi: 10.1007/s11886-0120255-0.

16. Pignatelli RH, McMahon CJ, Dreyer WJ, Denfield SW, Price J, Belmont JW, Craigen WJ, Wu J, El Said H, Bezold LI,
Clunie S, Fernbach S, Bowles NE, Towbin JA (2003) Clinical characterization of left ventricular noncompaction in children: a relatively common form of cardiomyopathy. Circulation, 108: 2672-2678. doi: 10.1161/01. CIR.0000100664.10777.B8.

17. Sen-Chowdhry S, McKenna WJ (2008) Left ventricular noncompaction and cardiomyopathy: cause, contributor or epiphenomenon. Curr Opin Cardiol, 23: 171-175. doi: 10.1097/HCO.0b013e3282fdc939.

18. Stanton C, Bruce C, Connolly H, Brady P, Syed I, Hodge D, Asirvatham S, Friedman P (2009) Isolated left ventricular noncompaction syndrome. Am J Cardiol, 104: 1135-1138. doi: 10.1016/j.amjcard.2009.05.062.

19. Stöllberger C, Blazek G, Winkler-Dworak M, Finsterer J (2009) Cardiac and neuromuscular implications of left bundle branch block in left ventricular hypertrabeculation/ noncompaction. Can J Cardiol, 25: e82-e85.

20. Wang L, Fan C, Topol SE, Topol EJ, Wang Q (2003) Mutation of MEF2A in an inherited disorder with features of coronary artery disease. Science, 302: 1578-1581. doi: 10.1126/science. 1088477. 